\section{Cahiers de Narratologie}

Analyse et théorie narratives

$11 \mid 2004$

Figures de la lecture et du lecteur

\title{
Quand voir c'est (faire) penser. Motivation des chaînes anaphoriques et point de vue
}

\section{Alain Rabatel}

\section{(2) OpenEdition}

\section{Journals}

Édition électronique

URL : http://journals.openedition.org/narratologie/21

DOI : $10.4000 /$ narratologie. 21

ISSN : 1765-307X

Éditeur

LIRCES

Référence électronique

Alain Rabatel, «Quand voir c'est (faire) penser. Motivation des chaînes anaphoriques et point de vue », Cahiers de Narratologie [En ligne], 11 | 2004, mis en ligne le 01 janvier 2004, consulté le 15 novembre 2019. URL : http://journals.openedition.org/narratologie/21 ; DOI : 10.4000/narratologie.21

Ce document a été généré automatiquement le 15 novembre 2019.

\section{(c) (i) (9)}

Cahiers de Narratologie - Analyse et théorie narratives est mis à disposition selon les termes de la licence Creative Commons Attribution - Pas d'Utilisation Commerciale - Pas de Modification 4.0 International. 


\title{
Quand voir c'est (faire) penser. Motivation des chaînes anaphoriques et point de vue
}

\author{
Alain Rabatel
}

1 Le point de vue narratif ${ }^{1}$ (PDV) ne saurait se comprendre à partir de la pure et simple transposition des règles de perspective des sémiotiques de l'image fixe ou mobile : en effet, il serait naif de croire analyser le PDV en ne recherchant dans les textes qu'un point de l'espace (analogue à celui occupé par le photographe, le peintre, le chef opérateur) à partir duquel les objets seraient perçus et structurés, en laissant croire que ce point (Genette parle de « foyer ») puisse être nettement isolable de l'objet perçu et indépendant de lui ${ }^{2}$.

2 En effet, lorsqu'on détermine les traces énonciatives du sujet percevant des textes de fiction à partir de la référenciation de ce qui est perçu et qu'on s'intéresse à l'articulation de la dimension phénoménologique des perceptions avec son expression linguistique, on constate que "percevoir ", n'est pas une source de savoir isolable des autres sources « évidentielles (emprunt à autrui, inférences à partir d'une perception confrontée à un savoir préexistant) : la perception n'est jamais un donné qui s'impose de lui-même à l'observateur, c'est toujours-déjà une construction intellectualisée, médiatisée par le langage (Vogeleer 1994). Cette donnée est confirmée par d'autres approches qui, à partir de cadres théoriques différents, aboutissent à des conclusions identiques :

La référence ne se définit [...] plus en fonction des entités du monde naturel (individus, objets, arguments) mais de l'expérience (noétique) qu'un sujet fait d'un certain donné phénoménal (noématique). Dès lors, la valeur spatio-temporelle de ce dernier, qui détermine l' " image mentale » associée à la représentation sémantique de l'énoncé, grâce à laquelle fonctionne ce qu'on appelle l' "impression référentielle" (Rastier 1991), se trouve étroitement liée aux valeurs modales, aspectuelles et autres de l'acte perceptivo-cognitif lui-même, soit au "point de vue » que l'énonciateur-observateur a sur le contenu événementiel de ses énoncés. [...] On énonce sa perception des événements constitutifs de la référence propre à ses énoncés, jamais les seuls objets perçus (Ouellet et alii, 1994 : 137). 
3 Ainsi, à l'instar de l'indissociabilité du recto et du verso de la feuille de papier, la construction linguistique des objets perçus et la construction linguistique du sujet percevant sont indissociables, voire interdépendantes, la référenciation des objets étant une des conditions (et un des garants) de la référenciation du sujet (et réciproquement). Nous vérifierons cette interdépendance, autrement dit cette double mimésis du sujet et de l'objet (Rabatel 2000a : 57), à travers l'intrication linguistique des perceptions et des pensées qui aboutit au cumul des valeurs descriptives et interprétatives (I). Ensuite nous mettrons en relief les valeurs épistémiques (ou cognitives), axiologiques, thymiques du PDV à propos des stratégies présidant à la constitution des chaînes anaphoriques dans la référenciation des référents évolutifs (II).

1 Valeurs descriptive et interprétative des perceptions représentées

4 A la différence de Genette, nous avons contraint la notion de PDV en nous intéressant à l'expression linguistique des perceptions, ce qui nous a amené à définir le PDV comme l'expression linguistique de perceptions représentées, le terme faisant système avec celui de pensée/parole représentée, pour le DIL (Bally 1912a, b et 1914). Il est certain que le PDV ne se limite pas aux comptes-rendus de perception ${ }^{3}$, a fortiori aux seules perceptions représentées. Mais il est stratégiquement intéressant de partir des perceptions, et plus particulièrement des perceptions en contexte narratif hétérodiégétique : d'abord parce que la limitation du phénomène en favorise la saisie ; ensuite parce que les caractéristiques de la représentation des perceptions remettent en question les oppositions (certes inévitables, mais à ne pas prendre au pied de la lettre) entre subjectivité et objectivité, ou, dans le domaine narratif, entre « interne » et «externe»; enfin parce que leur nature dialogique émerge aussi, sur un mode mineur, dans les contextes narratifs à la troisième personne, ce qui permet de mettre en valeur la spécificité de ces PDV perceptuels, comme formes pré-réflexives de la subjectivité (Banfield 1995: 293-313, Rabatel 2001a: 89-93), permettant ainsi de proposer un continuum, des formes pré-réflexives aux formes réflexives caractéristiques des discours rapportés.

5 Pour faciliter la compréhension de la nature polyphonique du PDV, il nous faut brièvement présenter le cadre théorique ducrotien à partir duquel nous appréhendons le PDV : conformément à Ducrot 1984, nous distinguons entre locuteur ${ }^{4}$ et énonciateur. Le locuteur (L) est l'instance qui profère un énoncé (dans ses dimensions phonétiques et phatiques ou scripturales, selon un repérage déictique ou selon un repérage indépendant d'ego, hic et nunc. L'énonciateur (E) est à l'origine d'un pdv. Proche du sujet modal de Bally, c'est l'instance des actualisations modales; c'est elle qui assume l'énoncé, en un sens nettement moins abstrait que la prise en charge découlant de l'ancrage déictique. Si, dans un énoncé monologique, le locuteur est aussi énonciateur, en revanche, dans un énoncé dialogique, il peut y avoir coexistence de plusieurs énonciateurs dans le discours d'un seul locuteur: ainsi, dans un énoncé ironique du type "On promenait dans les fiacres les vainqueurs de la Bastille, ivrognes heureux déclarés conquérants au cabaret », Chateaubriand, en tant que locuteur, met en scène un énonciateur second e $2^{5}$ qui admire les sans-culottes, et en tant qu'énonciateur premier (E1), il se distancie de toute source énonciative qui viendrait à partager cette admiration. Ainsi, cet énoncé comporte-t-il le jugement dépréciatif de L1/E1 envers un énonciateur second dont le jugement est rapporté, représenté, sans qu'il s'agisse d'un discours direct : e2 n'est pas locuteur (second), il est la source énonciative d'un point de 
vue dans une "phrase sans parole» (Banfield 1995). Autrement dit, si tout locuteur est toujours énonciateur, la réciproque n'est pas vraie.

6 En contexte narratif hétérodiégétique, dès que la perception a comme sujet ${ }^{6}$ un personnage, c'est ce dernier qui est la source du PDV, et non le narrateur ${ }^{7}$. Sur le plan énonciatif, les perceptions représentées coréfèrent à une origine énonciative distincte du locuteur-narrateur (L1/E1) : ce décrochage énonciatif est construit par l'opposition fonctionnelle des plans, comme on l'observe en (1) et en (2), et notamment par l'imparfait, dont la valeur d' "altérité énonciative » suppose « une dissociation de la source énonciative, mettant entre parenthèses le hic et nunc de l'énonciateur primaire au profit d'un ancrage sur le repère secondaire » (Mellet $2003: 90)$ :

(1) Zénon administra un réconfortant et examina la jambe ; les os en deux endroits sortaient de la jambe qui elle-même pendait en lambeaux. Rien dans cet accident ne ressemblait à l'effet de ruades; les marques de sabots n'étaient nulle part visibles (Yourcenar, L'œuvre au Noir Folio : 257).

2) La meute contourna silencieusement un massif rocheux couvert de ronces et se dirigea vers un chêne à l'énorme tronc noueux. Orientant sans cesse ses oreilles, différenciant les bruits, situant leur origine et leur éloignement, Bien-Noir entendit presque simultanément un campagnol s'enfuir à toutes pattes sur le sol feuillu, les griffes d'un écureuil remontant précipitamment en haut de son arbre, le chuintement d'une limace rampant non loin sur un champignon, les grattements d'un blaireau en train d'agrandir son terrier à trente pas d'ici, le minuscule crissement des dents d'un loir adulte entamant la base du champignon sur lequel évoluait la limace (Folco, Un loup est un loup Points Seuil 327s).

7 Certes, l'altérité énonciative peut dissocier le locuteur/énonciateur hic et nunc et le locuteur/énonciateur en une époque $\mathrm{X}$ du passé, mais la dissociation peut renvoyer à deux sources ontologiquement différentes, comme c'est le cas en (1) et (2), le procès perceptuel à l'imparfait renvoyant au sujet de l'énoncé en position saillante, et non au locuteur sujet de l'énonciation.

8 Sur le plan textuel, en (1) et en (2), le procès de perception prédiqué dans les premières propositions de premier plan, se développe dans les propositions suivantes, qui construisent le second plan : la progression thématique montre que la perception est associée à des procès mentaux successifs qui sont comme la concrétisation de l'examen détaillé auquel Zénon (ou le loup) se livre(nt), ces mouvements perceptifs-interprétatifs coréférant à la vision de l'énonciateur-focalisateur, et non au récit du narrateur anonyme.

9 Sur le plan sémantique, les perceptions représentées dans les deuxièmes plans entretiennent avec la perception prédiquée dans le premier plan une relation anaphorique méronomique (de type partie-tout): la perception prédiquée est linguistiquement présentée comme un tout, et se développe ensuite par l'aspectualisation ${ }^{8}$ des parties de ce tout. Celles-ci sont sous la dépendance sémantique du tout, y compris en l'absence de marque de subordination syntaxique, comme on le voit en (1), tant dans la première phrase que dans la suivante.

Sur le plan syntaxique, le procès prédiqué dans les premiers plans, le plus souvent au PS, correspond à une visée globale (et à une appréhension intellectuelle de l'événement, selon les analyses de Damourette et Pichon), cependant que l'aspectualisation des parties/moments de cette perception, dans les seconds plans, le plus souvent à l'IMP (mais on peut trouver d'autres formes, tels les participes présents de (2)), correspond à une visée sécante, propre à l'expression du décrochage énonciatif : 
c'est à ce niveau qu'intervient la notion de représentation, signifiant que le déploiement des perceptions est inextricablement associé à des mouvements de pensées. Ainsi, en (1), les perceptions successives sont à interpréter comme autant d'observations cliniques successives et de déductions qui, de proche en proche, contredisent les raisons alléguées par ceux qui ont demandé à Zénon de s'occuper du blessé : c'est ce qu'indiquent les connecteurs ajoutés en (1b), qui ne font qu'expliciter les mouvements déductifs motivant l'enchaînement des perceptions et des pensées (Rabatel 2001b) :

(1b) Zénon administra un réconfortant et examina la jambe ; les os en deux endroits sortaient de la jambe qui elle-même pendait en lambeaux. [Mais/or/cependant...] Rien dans cet accident ne ressemblait à l'effet de ruades ; [en effet/car/de fait...] les marques de sabots n'étaient nulle part visibles.

(1b) permet de dire que les perceptions représentées cumulent valeur descriptive (d'un état de fait) et valeur interprétative (l'état de fait étant appréhendé par le sujet dans son rapport au sujet), ce qui explique que les descriptions soient aussi des arguments co- ou anti-orientés (Rabatel 1999 et 2001b).

11 Certes, cette interprétation est directement tributaire du sémantisme du verbe, et, en amont, de la situation, qui implique une perception intentionnelle d'un agent humain ou, plus généralement, animé, comme c'est le cas avec l'auscultation d'un malade ou la traque du gibier. Certes encore, l'intentionnalité varie selon le sémantisme des verbes de perception et elle est plus importante avec les verbes de perception inférentielle ou représentationnelle (cf. Benzakour 1990). Mais même les verbes expérienciels dénotant une perception sensorielle directe qui, selon Benzakour, sont les verbes les moins chargés d'intentionnalité, n'excluent pas cette dimension interprétative. C'est ce que montre (2): entendre des bruits, les nommer en les référant à leur source (un campagnol, un écureuil, une limace ou un blaireau), c'est toujours-déjà interpréter. Cette réalité existe au niveau du lexique et elle est amplifiée par la syntaxe (Franckel et Lebaud 1990) : c'est ainsi que "voir X » est moins intentionnel que "voir si X », « voir que $\mathrm{X}$ », « voir comment X », ou " voir X qui » (les perceptions de (2) relevant de cette dernière tournure syntaxique). En réalité, l'intrication des valeurs descriptive et interprétative dépend certes du sémantisme intrinsèque du verbe de perception, mais découle surtout de l'opposition fonctionnelle des plans et du débrayage énonciatif :

(3) P1 Mais il y eut encore une volée de cloche, P2 et cette fois c'était bien le départ.

P3 On avait deux minutes (Zola, Lourdes François Bernouard, Paris 1929 : 63).

C'est ce qui se passe en (3): malgré l'existence d'un procès de perception et d'un verbe bien peu intentionnels, P2 et P3 correspondent aux pensées du prêtre, qui interprète sa perception comme un signal de départ imminent. Le fait que ces perceptions soient imbriquées à des fragments de DIL, ou, comme on l'a vu dans Rabatel 2001c, soient associées, dans le co-texte amont ou aval immédiat à des fragments de DD est un indice de plus en faveur de l'interprétation de ces énoncés descriptifs comme des PDV (et aussi de leur dialogisme pré-réflexif), mais c'est là un argument supplémentaire, et facultatif, pour ainsi dire, car l'interprétation serait la même si ces fragments étaient absents. Fondamentalement, en effet, ce qui justifie l'interprétation en terme de PDV, c'est le décrochage énonciatif, ainsi que le mécanisme inférentiel organisant sémantiquement cette suite d'énoncés comme des « phrases sans parole ».

La perception représentée est donc toujours (plus ou moins) associée à des procès mentaux qui la précèdent, l'accompagnent, la suivent, en écho ou en contrepoint, et ce 
y compris dans les cas où le mouvement perceptif semble à première vue dénué d'enjeux interprétatifs :

(4) Le père abbé resta un moment silencieux, regardant droit devant lui d'un air songeur, comme s'il hésitait non sur le contenu même de la réponse, mais sur l'opportunité de la donner. Le chemin de l'Etang s'incurvait au sud et, descendant en pente douce vers la pièce d'eau située en contrebas du monastère, longeait à présent la bordure orientale des vergers dont on apercevait les limites dans toutes les directions (Rio, Le perchoir du perroquet, Points Seuil 43).

En (4), le sujet percevant, explicitement mentionné par son nom propre, est à l'origine d'un procès de perception prédiquée dans la première phrase, et développée dans la seconde phrase, qui forme un second plan relativement à la première. L'usage de l'IMP, dans la deuxième phrase, donne à cette perception une coloration subjective : certes, le chemin « longe » l'étang depuis longtemps, mais cette information vient au lecteur par le filtre perceptif du père abbé, comme le signifie le marqueur temporel «à présent $»^{9}$ : le lieu est situé en référence aux linéaments de la pensée du religieux. La deuxième phrase n'est donc pas une notation descriptive objective émanant du narrateur, elle correspond à la perception motivée du père abbé, et elle est saturée d'une intentionnalité sous-jacente: comme si le regard que le père abbé portait sur les vergers était un prétexte pour retarder sa réponse (et pour faire accepter ce suspens à son compagnon de promenade, Frère Joachim). Dans ce contexte, l'indéfini «on » signale que cette perception est non seulement celle de l'abbé, mais encore celle de son compagnon de promenade, Frère Joachim ${ }^{10}:$ aussi longtemps que Joachim partage cette perception, il fait comprendre au père abbé que sa méditation est comprise, respectée.

En sorte qu'un énoncé en apparence aussi platement descriptif que la deuxième phrase de (4) ne se limite pas à la simple perception, et est surchargé de mouvements mentaux divers. A l'autre bout, (5) illustre une perception intentionnelle exprimant à un haut degré l'intrication d'un procès perceptif et de procès mentaux :

(5) Angelo retrouva la chambre du pavillon avec une agitation extrême. On avait remplacé le petit secrétaire et les bibliothèques par une grande table Henri II et des fauteuils très confortables. Angelo se promena de long en large avant de retrouver le calme. Il n'y avait plus de trace de parfum (Giono, Angelo Folio 217).

16 Ainsi l'expression linguistique de la perception mêle procès perceptifs et procès mentaux. Cette formule signifie que les perceptions sont souvent motivées par des pensées, ou prolongées par elles (ou réciproquement): la grande majorité des prédications de perception, et toute aspectualisation des perceptions surimposent perception et pensées ${ }^{11}$. Ces mécanismes constitutifs des mondes de la fiction et, plus généralement, des univers référentiels, reposent sur l'indissolubilité du lien entre processus énonciatif et expérience perceptive, comme le confirme l'analyse de la référenciation des référents évolutifs.

2 Les interactions percevoir/concevoir/juger dans la constitution des chaînes anaphoriques des référents évolutifs

La notion de référent évolutif concerne une entité subissant des transformations, susceptibles d'affecter (ou non) l'entité dans sa permanence ontologique : ainsi de la description des transformations d'une larve («l'embryon devient un têtard mangeur de très petites proies »). On peut aller jusqu'à inclure dans cette problématique la référenciation variable d'un même référent lorsque cette variabilité ne tient pas aux métamorphoses internes du référent (comme lorsque l'embryon se change en têtard 
avant de devenir une grenouille), mais est tributaire des modes variés d'appréhension du référent d'abord inconnu ou méconnu, avant d'être reconnu ${ }^{12}$.

Le mode de donation des référents évolutifs exprime tantôt le point de vue du locuteur/énonciateur premier, tantôt celui d'un énonciateur second distinct de L1/E1. Dans les chaînes anaphoriques, les lieux privilégiés où se marque ce PDV portent d'une part sur les anaphores grammaticales, d'autre part sur les anaphores lexicales. Ces deux grandes catégories d'anaphores entrent en jeu pour présenter les états de choses et pour préciser d'emblée, indissociablement, dans quel état d'esprit le locuteur ou l'énonciateur envisagent ces états de choses. C'est pourquoi il serait erroné d'interpréter le couple percevoir/concevoir comme des contraires et comme l'homologue du couple objectivité/subjectivité, ou du couple "interne »/ »externe » (Rabatel 1997) car l'objectivité n'est pas du côté du percevoir (« externe »), pas plus que la subjectivité du côté du concevoir (« interne »).

19 Commençons par quelques exemples d'anaphores grammaticales exprimant le PDV. Ainsi, un SN indéfini peut indiquer le PDV en signalant une première saisie d'un référent, ou encore, dans le cas de référents évolutifs, un savoir limité si la source énonciative manifeste qu'elle n'accède qu'à une partie des transformations (cf. les travaux de $\mathrm{M}$. Charolles et $\mathrm{C}$. Schnedecker sur les référents évolutifs, à qui nous empruntons les deux exemples suivants, ou encore ceux d'Achard-Bayle 2001) :

(6) Elle [ = la Belle ] se retourna vers sa chère Bête, dont le danger la faisait frémir. Quelle fut sa surprise ! La Bête avait disparu, et elle ne vit plus à ses pieds qu'un prince plus beau que l'amour. Il la remerciait d'avoir fini son enchantement (Mme Leprince de Beaumont, La Belle et la Bête).

Le référent est présenté sous la forme d'une entité inédite, la Belle étant re-catégorisée par une nouvelle dénomination et précédée par un déterminant indéfini, qui bloque toute référence à l'état antérieur, et traduit la nature bornée du PDV. La variation des anaphores permet par conséquent de signaler au lecteur si le focalisateur établit les connexions reliant les états entre eux. C'est ce que vérifient (7) et (8) :

(7) « On m'a assuré encore, dit le Chat, mais je ne saurais le croire, que vous aviez le pouvoir de prendre la forme des plus petits Animaux, par exemple de vous changer en un Rat, en une Souris ; je vous avoue que je tiens cela tout à fait impossible.

- Impossible ? reprit l'Ogre, vous allez voir », et, en même temps il se changea en une Souris, qui se mit à courir sur le plancher. Le Chat ne l'eût pas plus tôt aperçue qu'il se jeta dessus et la mangea (Perrault, Le Chat Botté, in Charolles 1993 b : 211).

(7b) «On m'a assuré encore, dit le Chat, mais je ne saurais le croire, que vous aviez le pouvoir de prendre la forme des plus petits Animaux, par exemple de vous changer en un Rat, en une Souris; je vous avoue que je tiens cela tout à fait impossible.

- Impossible ? reprit l'Ogre, vous allez voir ", et, en même temps il se changea en une Souris, qui se mit à courir sur le plancher. Le Chat ne l'eût pas plus tôt aperçu qu'il se jeta dessus et le mangea.

(7c) «On m'a assuré encore, dit le Chat, mais je ne saurais le croire, que vous aviez le pouvoir de prendre la forme des plus petits Animaux, par exemple de vous changer en un Rat, en une Souris; je vous avoue que je tiens cela tout à fait impossible.

- Impossible ? reprit l'Ogre, vous allez voir ", et, en même temps il se changea en une Souris, qui se mit à courir sur le plancher. Un chat entra alors dans la pièce. Il n'eût pas plus tôt aperçu l'Ogre métamorphosé qu'il se jeta dessus et le mangea.

(7d) « On m'a assuré encore, dit le Chat, mais je ne saurais le croire, que vous aviez le pouvoir de prendre la forme des plus petits Animaux, par exemple de vous 
changer en un Rat, en une Souris; je vous avoue que je tiens cela tout à fait impossible.

- Impossible ? reprit l'Ogre, vous allez voir ", et, en même temps il se changea en une Souris, qui se mit à courir sur le plancher. Un Chat entra alors dans la pièce. Il ne l'eût pas plus tôt aperçue qu'il se jeta dessus et la mangea.

Les diverses anaphores grammaticales (ou lexicales), en caractères gras, montrent que les énoncés renvoient à un sujet percevant doté de plus ou moins de savoir. (7) adopte le PDV du Chat, ce dont rend compte le fait que l'Ogre est présenté sous les traits de la souris: le Chat, qui a assisté à la métamorphose de l'ogre, réfère à ce dernier par l'évocation du dernier état du processus transformateur dont il a été témoin. C'est également le cas en $(7 b)$ : on peut même affirmer que le texte témoigne dans ce dernier cas d'un PDV du Chat plus étendu qu'en (7), puisque la référence à l'état antérieur de l'Ogre, avant sa transformation en souris, indique que le chat a bien conscience que la Souris qu'il a sous les yeux correspond bien à une métamorphose de l'Ogre. En (7c), nous sommes face à deux chats, celui qui entre dans la pièce ("un Chat») étant différent de celui qui a posé la question à l'Ogre : du fait que le texte mentionne "l'ogre métamorphosé ", nous voyons que ce dernier adopte le point de vue d'un narrateur qui a un savoir moins borné que celui du chat de (7d).

La référenciation ambiguë des objets par le choix d'anaphores fidèles ou infidèles confirme que le PDV ne se limite pas à la perception, et intègre toujours-déjà, en quelque manière, le savoir et les jugements que le locuteur ou l'énonciateur porte sur le référent :

(8) Grimaud vint ouvrir les yeux bouffis de sommeil. D'Artagnan s'élança avec tant de force dans l'antichambre qu'il faillit le culbuter en entrant.

Malgré le mutisme habituel du pauvre garçon, cette fois, la parole lui revint.

-Héla, là ! s'écria-t-il, que voulez-vous, coureuse ? que demandez-vous, drôlesse ?

D’Artagnan releva ses coiffes et dégagea ses mains de dessous son mantelet; à la vue de ses moustaches et de son épée nue, le pauvre diable s'aperçut qu'il avait affaire à un homme (A. Dumas, Les trois mousquetaires).

Les changements intervenus dans la chaîne de référence ("drôlesse " "un homme ») témoignent que Grimaud enregistre les modifications survenues: mais si Grimaud comprend progressivement que cette "drôlesse » n'est pas une femme, il ne comprend pas que l'homme est son maître. Aussi la dernière phrase de Dumas mêle-t-elle des logiques empathiques distinctes: d'une part, la dénomination de D'Artagnan est assumée par le narrateur, et lui seul ; les anaphores possessives ("ses coiffes", « ses mains ", "ses moustaches " et "son épée ») sont interprétables en relation à une logique empathique qui épouse la perspective du narrateur. Mais les mêmes possessifs peuvent être interprétés d'après la perspective limitée de Grimaud, et acquièrent de ce fait une valeur cataphorique relativement à « un homme ». Bien évidemment, les deux lectures sont complémentaires, et c'est leur confrontation qui est une des sources du plaisir de la lecture de ces récits où le lecteur, par le biais des simulacres de la fiction secondaire (Vuillaume 1990), est à la fois dans le vif de l'action et dans la fabrique du récit. Ainsi la phrase est-elle organisée autour de deux perspectives, à partir d'un point d'ancrage initial («D'Artagnan», pour la lecture anaphorique-savante) et final («un homme ", pour la lecture cataphorique-bornée). Au demeurant, le récit établit de luimême une hiérarchie entre ces deux lectures, comme l'indique la position dominée de ce "pauvre garçon", de ce "pauvre diable», la désignation de Grimaud par la réitération des descriptions définies rappelant la supériorité cognitive du lecteur, du fait qu'il suit la perspective du narrateur depuis le début de l'extrait. 

selon que le regard du locuteur évolue, offre, sur le plan linguistique, des arguments supplémentaires en faveur de l'intrication linguistique du percevoir et du concevoir. Le PDV narratif est donc un pluri-système sémio-linguistique combinant des systèmes sémiotiques qui jouissent d'une autonomie relative à l'égard de la langue, fonctionnant comme une sorte de pré-construit. Ces systèmes sémiotiques sont en nombre infini; relativement à notre propos, les plus fréquemment convoqués concernent les domaines qui organisent la vie sexuelle, sentimentale et affective, les relations culturelles, religieuses, idéologiques, sociologiques, politiques, économiques, etc. Toutes les données qui les composent relèvent des connaissances du monde, lesquelles ne sont pas que la somme de données factuelles, mais consistent aussi en cadres, grilles ou scénarios organisant la (pré-)compréhension du monde. Ces scénarios sont donc importants au titre de la dimension cognitive/épistémique du PDV. Sur le plan textuel, le déploiement de cette dimension épistémique n'est jamais aussi patent que lorsque le PDV prend la forme d'un compte-rendu progressif, à l'instar de ce qui se passe dans les plans de texte structurant la description comme parcours, ou programme: organisée selon une «temporalité » propre, la description focalisée par un sujet percevant mène de pair transformation sémiotique et construction de savoir en étant sans cesse soumise à des réajustements opérés par l'énonciateur, comme le montre (8), sous une forme amusée ${ }^{13}$.

En tant que pluri-système sémio-linguistique, le PDV ne se limite pas à l'analyse du dit, (contenu propositionnel concernant l'objet perçu); il comprend aussi les données relatives au dire et renvoie ainsi à l'ethos ${ }^{14}$ du sujet percevant: la référenciation de l'objet perçu fournit des indications précieuses sur le PDV du locuteur ou d'un énonciateur distinct de lui, c'est-à-dire sur les cadres de pensées, systèmes, représentations, passions, émotions qui structurent sa perception (et qui sont fortifiés ou transformés par elle).

Ainsi, le PDV ajoute aux valeurs épistémiques précédentes des valeurs axiologiques, idéologiques, thymiques, qui sont bien évidemment très importantes pour l'interprétation, et notamment pour l'interprétation de valeurs qui, du fait qu'elles n'apparaissent pas dans des jugements ou des commentaires explicites, semblent à première (et courte) vue « objectives ». Or rien de plus dangereux que d'être la dupe de ces illusions naïves; à partir de tout autres préoccupations, l'historien M. Bloch ne disait pas autre chose : "Un témoin a besoin d'un état civil. Avant même de faire le point sur ce que j'ai pu voir, il convient de dire avec quels yeux je l'ai vu» (M. Bloch L'étrange défaite Folio : 30).

Trois derniers exemples mettront en lumière cette dimension bidimensionnelle des perceptions, renseignant autant (sinon plus) sur l'objet perçu que sur le sujet percevant ${ }^{15}$. (9) illustre cette thèse à partir de l'analyse des anaphores lexicales :

(9) Le prince Chigi aurait pu parler certes longtemps, Sarrasine ne l'écoutait pas. Une affreuse vérité avait pénétré dans son âme. Il était frappé comme d'un coup de foudre. Il resta immobile, les yeux attachés sur le prétendu chanteur (Balzac, Sarrazine).

Barthes, commentant cet extrait dans S/Z, s'étonnait de cette formulation, «le prétendu chanteur » car Sarrazine sait que Zambinella est effectivement un chanteur... mais ignore que c'est un castrat :

Cahiers de Narratologie, 11 | 2004 
29 La formulation est énigmatique : on attendrait plutôt «la prétendue chanteuse », car dans la Zambinella, ce n'est pas le chant qui est une imposture, c'est le sexe, et ce sexe étant ici masculin (seul genre dont la langue dispose pour nommer le castrat), il ne peut être « prétendu » mais peut-être est-ce toute la personne de Zambinella qui est frappée de prétention, de fausseté, d'imposture, quelle que soit son apparence (Barthes, $S / Z$, fragment $473: 190$ ).

Cette interprétation révèle que Barthes réagit ici en lecteur abstrait et ne cherche pas à se mettre à la place du personnage: car, à prendre au sérieux la désignation de Zambinella, on constate que Sarrasine n'accepte pas que Zambinella soit autre chose qu'une femme. Voilà comment une anaphore en dit plus long qu'un long discours sur les motivations secrètes de Sarrasine...

31 De même, (10) exprime on ne peut plus nettement chez Zénon l'ethos du savant, par le biais des anaphores lexicales ou grammaticales, ainsi que par les degrés de modalisation qui entourent certains procès de perception :

(10) Vers le midi, il s'endormit couché à plat ventre dans un creux du sable, la tête contre le bras, sa loupe tombée de sa main reposant sous lui sur une touffe sèche. Au réveil, il crut apercevoir contre son visage une bête extraordinairement mobile, insecte ou mollusque qui bougeait dans l'ombre. Sa forme était sphérique; sa partie centrale, d'un noir brillant et humide, s'entourait d'une zone d'un blanc rosâtre ou terne; des poils frangés croissaient sur la périphérie, issus d'une sorte de molle carapace brune striée de crevasses et bossuée de boursouflures. Une vie presque effrayante habitait cette chose fragile. En moins d'un instant, avant même que sa vision pût se formuler en pensée, il reconnut que ce qu'il voyait n'était autre que son œil reflété et grossi par la loupe, derrière laquelle l'herbe et le sable formaient un tain comme celui d'un miroir. Il se redressa tout songeur. Il s'était vu voyant ; échappant aux routines des perspectives habituelles, il avait regardé de tout près l'organe petit et énorme, proche et pourtant étranger, vif mais vulnérable, doué d'imparfaite et pourtant prodigieuse puissance, dont il dépendait pour voir l'univers (Yourcenar, L'œeuvre au Noir Folio : 242).

32 -(10) met en scène deux visions successives, la première sacrifiant au jeu des apparences trompeuses, sans y céder tout à fait, comme l'indique la modalisation du procès de perception, la seconde étant à la fois une perception adéquate à l'objet et une mise en relation de l'objet perçu avec des représentations. La référenciation est, en elle-même, hautement significative : le premier procès de perception s'accompagne d' "assimilations " ${ }^{16}$, et laisse libre cours à une métaphore filée (en italiques), support d'une observation complaisamment délirante (cette vie "presque » effrayante est perçue avec une distance qui court depuis la modalisation initiale sur "apercevoir»). En revanche, la deuxième perception est annoncée par une anaphore conceptuelle («ce qu'il voyait»), à la source d'une démarche plus analytique pour l'appréhension de l'objet. Bref, les procès perceptifs sont ici mis en scène, d'une part en donnant à voir un mode d'appréhension distancié et intellectualisé, d'autre part en donnant à voir une appréhension immédiate, imagée, rapidement questionnée, en sorte que Zénon se donne à voir "voyant/cédant aux illusions des sens", ou "voyant/avec les yeux de l'âme »...

33 Enfin, les variations des anaphores grammaticales à l'intérieur du PDV d'Ali, en (11), témoignent non d'un changement de source du point de vue mais d'un changement d'attitude à l'égard des objets, en fonction des différents univers mentaux à l'intérieur desquels ces objets sont considérés : 
(11) Tout lui rentre à la fois dans les yeux : les teintes sombres d'un tapis, là on dirait une forêt mais faite d'arbres inconnus d'Ali, et là une gazelle peut-être, il n'a pas le temps d'en décider... la frange des nappes, le brun des bottes qui luit dessous, les tables si hautes que chaque Blanc a besoin d'une sorte de trône pour être à leur niveau, et les mains si pâles contre l'argent des couverts... A quoi peuvent bien servir ces fourches minuscules et ces poignards si courts? A quoi, sinon à égorger Ali ?... Et la rangée des barbes du blond au noir, de temps en temps un coup nu, une femme... Des femmes! A table avec des hommes! (Salvaing, Pays conquis Laffont $1977: 33)$ valeur métaphorique : «fourches » et «poignards » anaphorisent « couverts ». De plus, l'anaphore démonstrative indique une interprétation particulière de ces deux objets-là, relativement aux autres objets du discours; or l'idée d'une valeur déictique intratextuelle (d'une sorte d'hypotypose) résiste mal à l'analyse, dans la mesure où tous les autres objets du discours pourraient s'accommoder de ces mêmes anaphores, ce qui n'est pas le cas. En sorte qu'il faut alléguer ici une explication en terme d'univers de discours ${ }^{17}$ : en (11), la saisie par le démonstratif indique que le texte passe d'une appréhension descriptive factuelle, où dominait l'étonnement, à un mouvement « interprétatif », servant à l'expression d'une pensée représentée où domine la crainte. Autrement dit, la scène passe des perceptions étonnées et scandalisées d'Ali (anaphores définies ou indéfinies), à l'évocation inquiète des couverts inconnus, qui est l'occasion d'une interrogation (réitérée) sur le danger potentiel de ces objets inconnus pour la communauté d'Ali. Bref, les deux anaphores démonstratives signalent ce changement d'appréhension intellectuelle des objets, mais aussi la profondeur de la crise de confiance qui affecte les communautés.

Conclusion

Ainsi l'expression linguistique des perceptions, dès lors que celles-ci sont représentées, dénote que celles-ci sont étroitement imbriquées à des pensées. C'est pourquoi les perceptions représentées renseignent autant sur l'objet perçu que sur le savoir, l'axiologie, l'idéologie, voire les passions ou les émotions du sujet percevant, telles qu'elles structurent ses perceptions en amont même de celles-ci, en tant que cadre de pensée, ou telles qu'elles la structurent "on line", au cours même du processus scriptural, traversé par les réactions du sujet face à l'objet (de discours) représenté. En ce sens, la construction de l'objet et celle du sujet sont interdépendantes, et se conditionnent réciproquement.

À ce titre, il est légitime de parler d'effet-PDV car l'expression du PDV, construite par les représentations et croyances de l'énonciateur, dirige les perceptions et représentations du lecteur. Le "paradoxe» est que cet effet est masqué : en effet, en contexte hétérodiégétique, le PDV, en tant que compte-rendu de perception, exprime des jugements, une axiologie, des passions sans passer par un discours explicite. C'est ce qui concourt à l'efficacité si singulière de ces PDV qui se donnent l'apparence de l'objectivité alors qu'ils ont une origine énonciative spécifique et que la subjectivité y est (plus ou moins, mais néanmoins toujours) marquée par les traces du sujet modal, indépendamment du ego, hic et nunc.

Ce dialogisme discret plaide bien évidemment en faveur d'une articulation entre PDV en contexte narratif et en contexte argumentatif (Rabatel 2004), surtout si l'on prend en compte le fait que les énonciateurs sujets percevants sont des créations du locuteur/ 
énonciateur premier, qui est responsable de part en part de la scénographie énonciative...

\section{BIBLIOGRAPHIE}

ACHARD-BAYLE G. 2001 Grammaire des métamorphoses. Référence, identité, changement, fiction. Duculot, Bruxelles.

AMOSSY R. 1999 « La notion d'ethos de la rhétorique à l'analyse du discours », Images de soi dans le discours, Amossy, R. (ed), 9-30. Delachaux et Niestlé, Lausanne, Paris.

APOTHELOZ, Denis 1998 «Eléments pour une logique de la description et du raisonnement spatial » La description Reuter, Y. (ed), 15-31. Presses Universitaires du Septentrion, Villeneuved'Ascq.

BALLY C. 1912 a « Le style indirect libre en français moderne, I », Germanische Romanische Monatschrift, fas. 4, 549-556.

BALLY C. 1912 b « Le style indirect libre en français moderne, II », Germanische Romanische Monatschrift, fas. 4, 597-608.

BALLY C. 1914 « Figures de pensée et formes linguistiques », Germanische Romanische Monatschrift, fas. 6, 405-422 et 456-470.

BANFIELD A. 1995 Phrases sans parole. Théorie du récit et style indirect libre. Le Seuil, Paris.

BENZAKOUR F. 1990 Les compléments de comptes-rendus de perception: quelques cas en français. Thèse de doctorat, Strasbourg II.

CHAROLES M. et SCHNEDECKER C. 1993a : «Coréférence et identité. Le problème des référents évolutifs », Langages 112, 106-126, Larousse, Paris.

CHAROLES M. et SCHNEDECKER C. 1993b : «Les référents évolutifs : points de vue ontologique et phénoménologique », Cahiers de linguistique française 14, 197-227. Université de Genève.

COHN, D. 1990 La transparence intérieure. Le Seuil, Paris.

De MULDER W. 1998 « Du sens des démonstratifs à la construction d'univers ", Langue Française 120, 21-32. Larousse, Paris.

De MULDER W. et TASMOVSKY-De RICK L. 1997 : « Référents évolutifs, syntagmes nominaux et pronoms », Verbum Tome XIX, 1-2, 121-136.

DUCROT O. 1984 Le Dire et le Dit. Minuit, Paris.

DUCROT O. 1989 Logique, structure, énonciation. Minuit, Paris.

GENETTE G. 1972 Figures III. Le Seuil, Paris.

GENETTE G. 1983 Nouveau discours sur le récit. Le Seuil, Paris.

FRANCKEL J.J. et LEBAUD D. 1990 : Les figures du sujet. A propos des verbes de perception, sentiment, connaissance. Ophrys, Gap, Paris. 
HAMON P. 1981 Introduction à l'analyse du descriptif. Hachette, Paris.

KLEIBER G. et alii 1997 La continuité référentielle. Recherches linguistiques 20, Klincksieck/ Université de Metz.

MELLET, S. 2003 «Imparfaits en contexte : les conditions de la causalité inférée », Langue française 138, 86-96.

NONNON E. 1998 « L'activité descriptive comme démarche d'investigation dans le cadre d'une construction de connaissances ", in La description, Reuter (ed), 85-104. Presses universitaires du Septentrion, Villeneuve-d'Ascq.

OUELLET P. EL ZAIM A. BOUCHARD H : 1994 « La représentation des actes de perception : le cas de paraître », Les Cahiers de praxématique 22 135-156, Université de Montpellier 3.

RABATEL A. 1997 Une histoire du point de vue. Klincksieck, Paris/Centre d'Etudes Linguistiques des Textes et des Discours. Université de Metz.

RABATEL A. 1998 La construction textuelle du point de vue. Delachaux et Niestlé, Lausanne, Paris.

RABATEL A. 1999 « Mais dans les énoncés narratifs : un embrayeur du point de vue et un organisateur textuel », Le Français moderne, vol. LXV, 1, 49-60.

RABATEL, A. 2000a « Valeurs représentative et énonciative du 'présentatif' c'est et marquage du point de vue », Langue française, 128, 52-73.

RABATEL A. $2000 \mathrm{~b}$ « Un, deux, trois points de vue ? Pour une approche unifiante des points de vue narratifs et discursifs », La lecture littéraire 4, 195-254. Klincksieck, Paris.

RABATEL, A. 2001a « Les représentations de la parole intérieure : monologue intérieur, discours direct et indirect libres, point de vue », Langue française 132, 72-93.

RABATEL A. 2001b « La valeur délibérative des connecteurs et marqueurs temporels mais, cependant, alors, maintenant, et dans l'embrayage du point de vue. Propositions en faveur d'un continuum argumentativo-temporel », Romanische Forschungen 113-2, 153-170.

RABATEL A. 2001c « Le point de vue et l'organisation du texte » in Quelles grammaires enseigner à l'école et au collège. Discours, genres, texte, phrase, Garcia-Debanc et alii (eds), 227-240. Delagrave/CRDP Midi-Pyrénées.

RABATEL A. 2001d « La valeur de « on » pronom indéfini/pronom personnel dans les perceptions représentées », L'information grammaticale 88, 28-32.

RABATEL, A. 2002 « Le point de vue, entre grammaire et interprétation. Le cas de 'on' », in Lire, écrire le point de vue, Rabatel, A. (ed), 71-101. Lyon, CRDP de Lyon.

RABATEL, A. 2003 « Les verbes de perception en contexte d'effacement énonciatif : du point de vue représenté aux discours représentés », Travaux de linguistique 46-1, 49-88.

RABATEL, A. 2004 Argumenter (autrement) en racontant. Duculot, Bruxelles (à paraître).

RASTIER F. 1991 Sémantique et recherches cognitives. Presses Universitaires de France, Paris.

VOGELEER S. 1994 « L'accès perceptuel à l'information : à propos des expressions un homme arrive/on voit arriver un homme », Langue française 102, 69-83.

VUILLAUME M. 1990 Grammaire temporelle des récits. Minuit, Paris. 


\section{NOTES}

1. Le PDV est un synonyme de la notion de focalisation (narrative) analysée en France par Genette 1972 et 1983. Le changement de dénomination, qui vise notamment à éviter les confusions avec le concept de focalisation discursive, entend signaler une rupture avec le paradigme genettien, tant sur le plan de la définition de la notion que sur celui de son découpage (notamment à propos de la focalisation zéro et de la focalisation externe) : sur ces questions, cf. Rabatel 1997, chapitres 3, 12 et 13 et Rabatel 1998, chapitres 1 et 3.

2. Cette idée peut être illustrée par les définitions « ontologisantes » du volume de savoir affecté par nature au narrateur (omniscience), au personnage (savoir limité, notamment par l'impossibilité d'accéder aux pensées des autres personnages), et à l'hypothétique foyer de la focalisation externe (savoir minimal réduit à l'enregistrement des actions, parmi lesquelles les paroles) : or le volume du savoir ne peut pas être prédéterminé a priori, il varie selon les genres, les œuvres, les moments et dépend de l'expression linguistique du savoir : cf. infra, l'analyse de (8).

3. Cf. Rabatel 1997 et 1998. Depuis ces travaux, nous nous sommes intéressés (Rabatel 2000b) à d'autres formes d'expression du PDV notamment dans le premier plan, avec des passés simples, sans que ces perceptions ne soient longuement détaillées. Ce type de PDV nommé PDV embryonnaire (ou raconté) s'apparente au discours narrativisé de Genette. Cela montre que les formes du PDV, en tant que compte-rendu de perception, ne se limitent pas au PDV représenté et que leur dialogisme ne doit pas les assimiler seulement au seul DIL : sur ce point, cf. Rabatel 2003. Cela étant, dans le cadre de cet article, on n'abordera que le PDV représenté.

4. Sans compter le sujet parlant, dont la définition ne fait pas débat, par rapport au locuteur et à l'énonciateur.

5. La minuscule et le chiffre indiquent le caractère second et subordonné de cet énonciateur. Il en va de même pour les locuteurs seconds (12), enchâssés dans le discours de L1/E1.

6. Un verbe de perception se rapporte le plus souvent au sujet percevant : ainsi « examina » en (1) et « entendit » en (2). Lorsque le procès perceptuel est sous-entendu, l'attribution du PDV repose sur un mécanisme inférentiel, comme en (3).

7. Cette distinction croise la distinction genettienne entre mode et voix : c'est le narrateur qui raconte, mais c'est le personnage qui voit (cf. Ducrot 1989 : 181). Il est certain que la distinction énonciative n'épuise pas le débat de la nature des liens sémantiques entre le locuteur premier et les énonciateurs seconds. Les relations entre L1/E1 et 12/e2 relèvent tantôt de la responsabilité ou de la non responsabilité, lorsque les relations sont explicites, tantôt de la consonance ou de la dissonance (Cohn 1990), lorsque ces liens sont implicites. Dans les deux cas, ces liens sont graduels. Dans l'exemple de Chateaubriand, les italiques signalent que L1/E1 n'est pas responsable du jugement ; dans l'exemple (1), en l'absence de marques explicites, et en vertu du pacte de croyance réaliste, le lecteur considère par défaut que $\mathrm{L} 1 / \mathrm{E} 1$ entérine comme vraie la perception de e 2 : il y a donc consonance. Il est évident qu'en l'absence de sujet percevant distinct de L1/E1, la perception représentée est prise en charge par le narrateur.

8. Au sens que les spécialistes de la description donnent à ce terme : l'aspectualisation correspond ici au déroulement des aspects (ou parties) d'un objet du discours d'abord évoqué comme un tout dans le « thème-titre » de la description. 
9. Il en va de même pour le marqueur spatial « ici » dans l'exemple de M. Folco : ce marqueur à valeur généralement déictique, comme « à présent », en (4), signale que l'énoncé auquel il appartient est à considérer comme renvoyant à une je-origine fictive, celle de l'énonciateur, et non comme relevant du narrateur anonyme. Il vise à donner aux énoncés de troisième personne, dans le cadre de l'énonciation historique, une dimension personnelle : c'est ce que Banfield 1995 appelle le paradoxe de la subjectivité, un « il » et un temps du passé pouvant équivaloir à un « je » et à un temps du présent.

10. Bref « on » est en réalité pronom personnel. Cf. Rabatel 2001d et 2002.

11. Cf. Ouellet et alii, $1994: 142-7$, à propos des figures schématisant les catégories de la description conceptuelle et linguistique.

12. Cette définition étendue des référents évolutifs peut être critiquée, mais il n'y a pas de mécanismes linguistiques différents dans les deux cas, cf. De Mulder et TasmovskyDe Rycck 1997.

13. Cf. Hamon 1981 : 109 ; Apothéloz 1998 ; Nonnon 1998 : 97ss notamment.

14. Cf. Amossy 1999.

15. Ouellet parle de double vision énonçante et énoncée (art. cité, 138);

16. Dans les textes descriptifs, il s'agit du processus, complémentaire de l'aspectualisation (métonymique), qui repose sur des relations de type métaphorique que telle partie du tout entretient avec des référents extérieurs à l'objet (du discours) décrit.

17. Cf. De Mulder 1998, in Langue française $120: 31$.

INDEX

Mots-clés : point de vue, référents évolutifs, dialogisme des perceptions, valeurs pragmatiques des perceptions

\section{AUTEUR}

\section{ALAIN RABATEL}

IUFM de Lyon, ICAR, UMR 5191, Université Lyon 2, CNRS, ENS-LSH. Alain Rabatel est Professeur de Sciences du Langage à l'IUFM de Lyon, et mène ses recherches dans le cadre du Laboratoire ICAR, UMR CNRS 5191, Université Lumière-Lyon 2. Dernier ouvrage paru : Argumenter en racontant (DeBoeck, novembre 2004), consacré aux formes d'argumentation liées au point de vue en contexte narratif et aux interactions lecture/ écriture dans le cadre de l'écriture d'imitation/d'invention. 